\title{
Esquema de engrossamento com agregação dupla de pares para o precondicionador Multigrid Algébrico
}

\author{
Henrique Gomes de Jesus \\ Maria Claudia Silva Boeres $\mathbb{N}^{2}$ \\ Lucia Catabriga $\beta^{3}$ \\ Universidade Federal do Espírito Santo
}

\begin{abstract}
Resumo. Este trabalho se destina à análise empírica do método multigrid algébrico (AMG) como precondicionador do método do Resíduo Mínimo Generalizado (GMRES) utilizando uma estratégia de engrossamento conhecida como agregação dupla de pares (DPA, do inglês - Double Pairwise Aggregation). Esta estratégia consiste em aplicar um algoritmo de matching em grafos duas vezes em cada nível da hierarquia a fim de produzir os operadores de restrição e interpolação. O esquema de engrossamento DPA é testado em um conjunto de matrizes do repositório de matrizes esparsas SuiteSparse Matrix Collection, relacionadas a variadas aplicações. O AMG como precondicionador, empregando tanto a técnica de engrossamento DPA quanto as clássicas, é comparado ao precondicionador ILU, ressaltando vantagens e desvantagens de cada escolha.
\end{abstract}

Palavras-chave. Métodos multigrid. Multigrid algébrico. Double Pairwise Aggregation. Métodos iterativos. Precondicionadores.

\section{Introdução}

Neste trabalho, utilizamos o Método do Resíduo Mínimo Generalizado (GMRES, do inglês): um método de solução de sistemas lineares assimétricos que aproxima a solução através do vetor de resíduo mínimo em um subespaço de Krylov [14. Embora os métodos não estacionários sejam mais robustos que os estacionários, por convergirem com menos iterações, cada iteração requer mais operações de ponto flutuante, e portanto, sua robustez pode ser ampliada ainda mais por meio do uso de técnicas de precondicionamento. Tais técnicas consistem em modificar o sistema original $A u=f$, transformando-o num sistema equivalente, ou seja, que possui a mesma solução, mas que é melhor condicionado, ampliando a taxa de convergência e diminuindo o número de iterações. No precondicionamento à esquerda, o sistema precondicionado pode ser representado por $M^{-1} A u=$ $M^{-1} f$, onde a matriz de precondicionamento $M$ deve ser próxima a $A$, mas suficientemente simples de se construir.

A cada iteração $i$, o método GMRES executa $i$ produtos escalares, $i$ combinações lineares e 1 produto matriz-vetor. Deste modo, a ação do precondicionador no método iterativo ocorre no produto matriz vetor $v=M^{-1} A z$, sendo calculado em dois passos: No primeiro o produto matrizvetor original é calculado $(w=A z)$ e no segundo o sistema linear trivial $M v=w$ é resolvido por algum método de baixa complexidade.

Já os métodos iterativos estacionários [14], ou relaxações, realizam uma sequência de iterações com um número bem menor de operações de ponto flutuante, entretanto podem convergir muito

\footnotetext{
${ }^{1}$ henrique.g.jesus@aluno.ufes.br

2 boeres@inf.ufes.br

3luciac@inf.ufes.br
} 
lentamente. Os métodos de relaxação podem ser melhorados através do emprego de uma estratégia conhecida como Multigrid 3, que transforma o problema em outro com menos variáveis, onde a execução do método de solução é mais barata. Chamamos esta nova versão do problema de "discretização grosseira", em oposição à original, dita "refinada". A solução encontrada no domínio grosseiro pode, por fim, ser transferida de volta ao domínio refinado original.

Os métodos Multigrid podem ser divididos em duas categorias: Multigrid Geométrico (GMG) e Multigrid Algébrico (AMG) [1. Enquanto cada método GMG é construído a partir de um conjunto de discretizações de um domínio específico, o AMG é uma generalização que busca solucionar sistemas lineares definidos por quaisquer matrizes, sem necessidade de que se conheça a geometria do problema a priori.

O AMG foi introduzido nos anos 80 [1], quando o princípio de Galerkin e as transferências entre discretizações baseadas em produtos matriz-vetor foram implementados no GMG com o objetivo de aumentar sua robustez [21. Na metade dos anos 90 o interesse pelo AMG se intensificou tanto pelo aumento da complexidade geométrica das aplicações, quanto pela necessidade de ser disponibilizado um sistema Multigrid comercial [16]. Nesta época foram publicadas referências importantes para o estudo e compreensão do AMG, das quais destacamos [3, 9, 17, 19.

A fim de produzir as matrizes que existirão na versão reduzida do problema é utilizado um esquema de engrossamento, que decide quais variáveis existirão na fase grosseira do problema. Diversas estratégias já foram desenvolvidas, como as estratégias clássicas propostas em [17], o Hybrid Modified Independent Set (HMIS), o Parallel Maximal Independent Set (PMIS) [15], as agregações suavizadas [20], a Compatible Relaxation [2] e a Agregação Dupla por Pares (DPA) [11], apenas para citar algumas.

Neste trabalho, pretendemos apresentar um estudo do método AMG, seu esquema de engrossamento DPA e seu uso como precondicionador, bem como resultados experimentais comparativos na solução de matrizes gerais. Assim, as seções são organizadas como se segue: na Seção 2 é introduzido o método AMG como precondicionador do GMRES empregando o esquema de engrossamento DPA, na Seção 3 são apresentados os resultados numéricos obtidos utilizando um conjunto de matrizes do repositório de matrizes esparsas SuiteSparse Matrix Collection 4 ] e, na Seção 4, é feita uma análise dos resultados, destacando vantagens e desvantagens em cada método testado.

\section{Precondicionador Multigrid Algébrico com DPA}

O método Multigrid Algébrico acelera a convergência de métodos iterativos transformando o problema em outro com menos variáveis. Quando isso ocorre, dizemos que o problema foi discretizado em um domínio mais grosseiro. Este procedimento pode ser aplicado recursivamente como ilustrado na Fig. 1, onde o nível 1 representa a discretização original, mais refinada, e os níveis 2 e 3 representam discretizações sucessivamente mais grosseiras. Deste modo, o problema pode ser solucionado no domínio mais grosseiro, onde a execução das iterações do método de solução é naturalmente mais barata, e a solução pode ser levada de volta ao domínio mais refinado 3. Tais estratégias se tornaram populares por, em tese, possuírem complexidade próxima da linear [12].

A transferência de um vetor do domínio refinado para o grosseiro é chamada de restrição e a transferência de um vetor do domínio grosseiro para o refinado é chamada de interpolação ou prolongação. Uma iteração do método Multigrid é caracterizada por sucessivas restrições e interpolações, que podem ser executadas em variados esquemas. Neste trabalho adotamos o esquema denominado Ciclo V. Nele, a solução é transferida até o domínio mais grosseiro e, então, de volta até o mais refinado, aplicando um número parametrizado de relaxações em cada nível. Ao utilizar o AMG como precondicionador, em cada produto matriz-vetor é executado um único Ciclo V do Multigrid no sistema $M v=w$ seguindo as ideias descritas em [21] e [8].

O processo de restrições e interpolações define uma hierarquia de discretizações dos dados de 


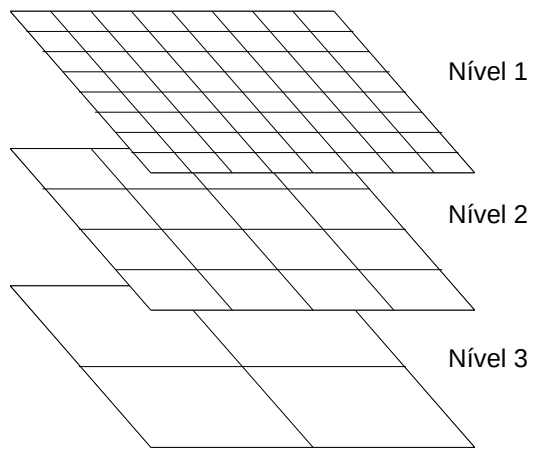

Figura 1: Hierarquia de discretizações.

um sistema. Considere o sistema original dado por $A^{h} u^{h}=f^{h}$ e a a versão grosseira da matriz $A^{h}$ obtida através de uma operação de Galerkin [7] dada por:

$$
A^{2 h}=R^{h} A^{h} P^{h}
$$

onde o índice sobrescrito $h$ de cada variável indica o nível (ou domínio) ao qual pertence na hierarquia de discretizações. $R^{h}$ e $P^{h}$ são os operadores de interpolação e restrição, com $R^{h}=$ $\left(P^{h}\right)^{T}$.

A utilização do método Multigrid em sistemas lineares definidos a partir de matrizes gerais advindas de aplicações diversas - demanda uma fase de pré-processamento chamada de setup, onde os operadores $R^{h}$ e $P^{h}$ são construídos. Nessa fase, é empregado um esquema de engrossamento entre níveis, que visa decidir quais variáveis serão criadas nos domínios mais grosseiros e suas relações de dependência com as variáveis dos domínios mais refinados [18].

As estratégias clássicas de engrossamento propostas por Stüben [17] se baseiam em aplicar heurísticas de agregação de vértices em grafos que representam a matriz de coeficientes do sistema linear. Quando se utiliza o esquema DPA [11, este processo é realizado através de um algoritmo de matching [6. A Fig. 2 ilustra um processo de matching e o engrossamento a ele correspondente.
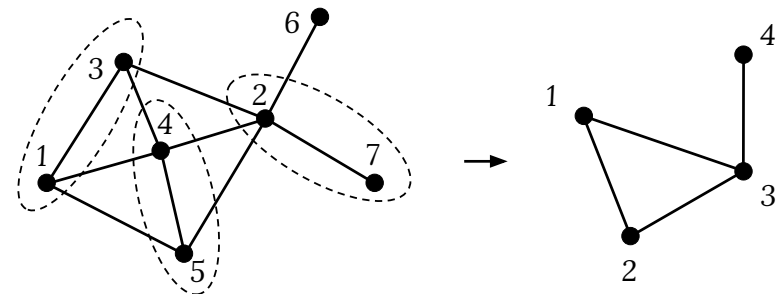

Figura 2: Matching sobre grafo à esquerda. As arestas selecionadas estão circuladas. Na matriz grosseira, cada aresta terá se tornado uma única variável, como representado à direita.

Em esquemas de engrossamento por agregação, as variáveis de um domínio mais refinado podem ser separadas em conjuntos disjuntos (agregados), associados a variáveis do domínio grosseiro. A fim de ampliar a redução da ordem do problema, o processo de matching é realizado duas vezes em cada nível, de forma que os agregados sejam formados por pares de pares de variáveis (vértices no grafo correspondente), elevando o potencial de redução da ordem do sistema linear em até quatro vezes [1]. Deste modo, o número máximo de agregados por variáveis do sistema grosseiro 
é constante e igual a quatro, enquanto que nos esquemas clássicos de engrossamento, cada variável do sistema grosseiro está associada a todos os vizinhos - cujo número não se conhece a priori - de uma variável diretamente correspondente no sistema refinado.

\section{Resultados Experimentais}

Os experimentos numéricos foram realizados com 17 matrizes do repositório de matrizes esparsas SuiteSparse Matrix Collection. Os resultados obtidos são analisados com respeito ao número de iterações e tempo computacional. Para cada matriz é definido um sistema linear trivial, cujo vetor dos termos independentes é calculado considerando que a solução exata é o vetor $(1,1, \ldots, 1)^{t}$ de ordem $n$. As principais características dessas matrizes estão descritas na Tabela 1. As colunas indicam, da esquerda para a direita, o índice de identificação da matriz (\#), nome (Nome), ordem (n), número de elementos não nulos (nnz), simetria (simétrica) e porcentagem de esparsidade (esparsidade (\%)). O asterisco $(*)$ indica que a matriz é diagonal dominante. Para cada matriz, foram testados os seguintes precondicionadores: ILU - Fatoração LU Incompleta 14]; Stb - AMG com esquemas de engrossamento clássicos de Stüben [17]; DPA-S - AMG com DPA e SOR (Método sobre relaxação sucessiva [14]) como relaxador; e DPA-G - AMG com DPA e GMRES como relaxador.

Tabela 1: Principais características das matrizes.

\begin{tabular}{|c|c|c|c|c|c|}
\hline$\#$ & Nome & $\mathbf{n}$ & nnz & simétrica & esparsidade (\%) \\
\hline 1 & rail_5177 & 5.177 & 35.185 & sim & 99,8687 \\
\hline 2 & aft01 & 8.205 & 125.567 & $\operatorname{sim}$ & 99,8135 \\
\hline 3 & FEM_3D_thermal1 & 17.880 & 430.740 & não & 99,865 \\
\hline 4 & Dubcova2 & 65.025 & 1.030 .225 & $\operatorname{sim}$ & 99,9756 \\
\hline 5 & $\mathrm{H} 2 \mathrm{O}$ & 67.024 & 2.216 .736 & $\operatorname{sim}$ & 99,9507 \\
\hline 6 & FEM_3D_thermal2 & 147.900 & 3.489 .300 & não & 99,9840 \\
\hline 7 & parabolic_fem & 525.825 & 3.674 .625 & $\operatorname{sim}$ & 99,9987 \\
\hline 8 & atmosmodj* & 1.270 .432 & 8.814 .880 & não & 99,9995 \\
\hline 9 & atmosmodd* & 1.270 .432 & 8.814 .880 & não & 99,9995 \\
\hline 10 & Serena & 1.391 .349 & 64.131 .971 & $\operatorname{sim}$ & 99,9967 \\
\hline 11 & Geo_1438 & 1.437 .960 & 60.236 .322 & $\operatorname{sim}$ & 99,9971 \\
\hline 12 & atmosmodl & 1.489 .752 & 10.319 .760 & não & 99,9995 \\
\hline 13 & af_shell10 & 1.508 .065 & 52.259 .885 & $\operatorname{sim}$ & 99,9977 \\
\hline 14 & G3_circuit & 1.585 .478 & 7.660 .826 & $\operatorname{sim}$ & 99,9997 \\
\hline 15 & Transport & 1.602 .111 & 23.487 .281 & não & 99,9991 \\
\hline 16 & Cube_Coup_dt6 & 2.164 .760 & 124.406 .070 & $\operatorname{sim}$ & 99,9973 \\
\hline 17 & Bump_2911 & 2.911 .419 & 127.729 .899 & $\operatorname{sim}$ & 99,9985 \\
\hline
\end{tabular}

Os experimentos foram executados no supercomputador Lobo Carneiro (LoboC) da UFRJ. O LoboC conta com 504 CPUs Intel ${ }^{\circledR}$ Xeon ${ }^{\circledR}$ E5-2670v3, totalizando 6048 núcleos de CPU, distribuídos em 252 nós computacionais com 64 GBytes de memória cada. O sistema operacional instalado no LoboC é o Suse Linux Enterprise [10]. Uma vez que o LoboC é uma máquina compartilhada, a carga varia ao longo da realizações dos testes, tornando os resultados instáveis. Devido a este fato, todas as execuções foram realizadas 5 vezes, eliminando os menores e os maiores valores encontrados e extraindo a média aritmética dos restantes em cada caso. Todos os 
códigos implementados neste trabalho foram escritos na linguagem de programação C, compilados usando o compilador $g c c$ versão 5.4 .0 com nível de otimização -O3 e estão disponíveis em https://github.com/mod-comp-ufes/AMG

Em todos os testes adotamos a tolerância de $\varepsilon=10^{-8}$ e o número máximo de iterações igual a 1.000 - qualquer matriz que dependa de mais de 1.000 iterações para convergir foi considerada não convergente. $\mathrm{O}$ fator de preenchimento $p$ do precondicionador ILU foi escolhido dentre os valores $p=0,1,2,3,4$. Os resultados apresentados para esse precondicionador variam de acordo com o valor de $p$ com o qual obteve-se o menor tempo computacional, uma vez que as matrizes são oriundas de aplicações diversas.

Nos precondicionadores Stb e DPA-S são consideradas duas relaxações do SOR com parâmetro de relaxação igual a 1, 5 e apenas um nível de engrossamento no Ciclo V. No caso do precondicionador DPA-G, que utiliza o relaxador GMRES, foram considerados $\log _{4} n-2$ níveis de engrossamento no Ciclo $\mathrm{V}$, sendo $n$ a ordem do sistema, com o objetivo de reduzi-lo a uma ordem trivial no nível mais grosseiro 5]. Testes preliminares utilizando essa mesma estratégia com Stb e DPA-S não produziram bons resultados.

O GMRES empregado como relaxador no precondicionador DPA-G utiliza uma base de Krylov com 10 vetores e apenas uma iteração GMRES, exceto no último nível, onde são consideradas no máximo 1.000 iterações GMRES e tolerância de $10^{-8}$. Assim, no nível mais grosseiro o método é iterado até atingir a tolerância (ou número máximo de 1.000 iterações), diferentemente do DPA-S, onde se executam apenas 2 iterações fixas nas etapas de restrição e interpolação.

A Tabela 2 apresenta para cada matriz e precondicionador Stb, DPA-S, DPA-G e ILU, o número de iterações do GMRES (Iter) e os tempos computacionais (Tempo), normalizados pelo maior tempo. Os testes que não convergiram são representados por $\dagger$.

Tabela 2: Normalização dos tempos de execução em segundos e número de iterações. Os melhores resultados dos tempos computacionais estão destacados em negrito para cada matriz.

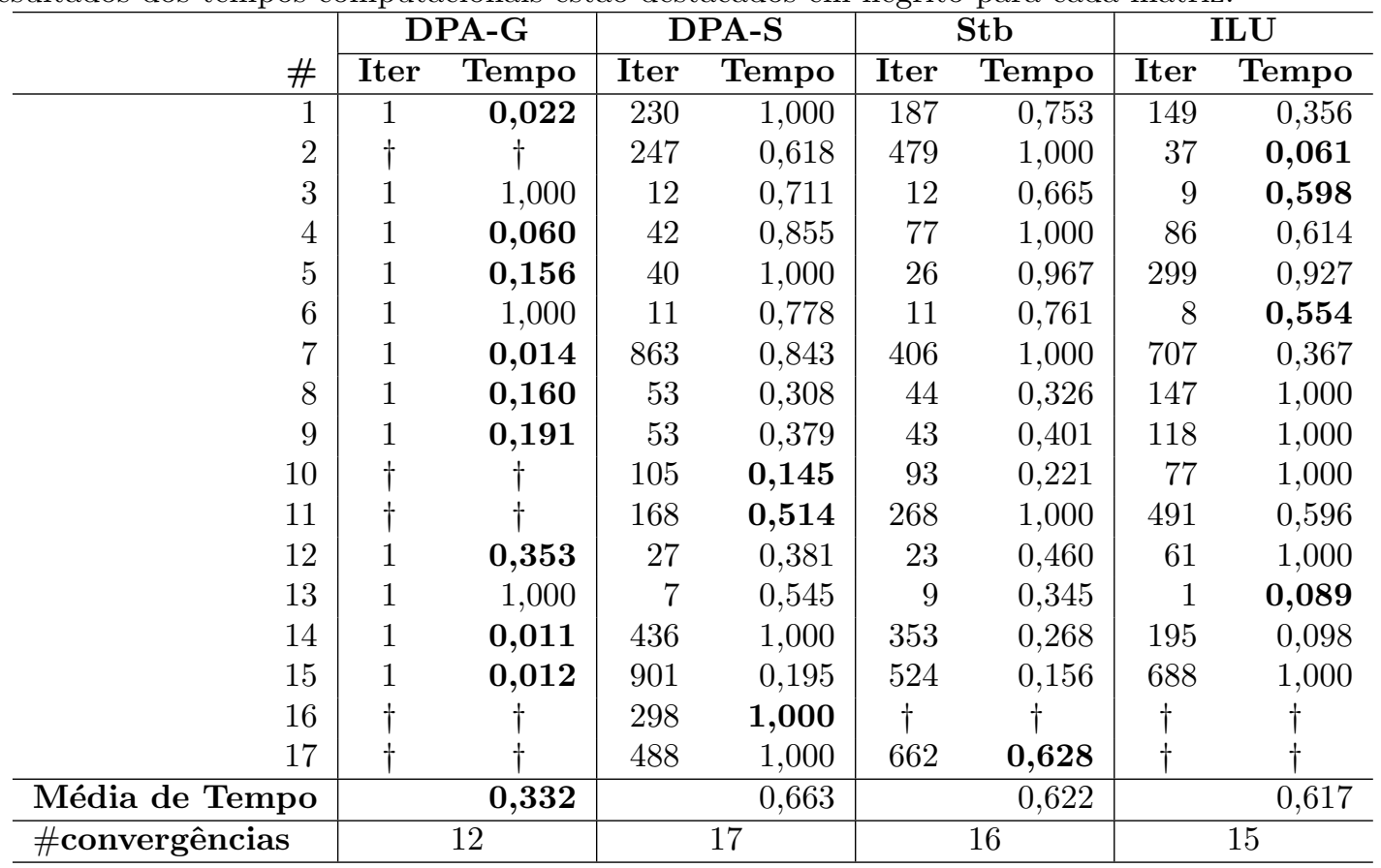


Para esse conjunto de matrizes o uso de precondicionamento é essencial, pois possibilitou o aumento da convergência de $23 \%$ para o GMRES sem precondicionamento - conforme relatado em [5] - para 100\% das matrizes para o GMRES com precondicionamento: o DPA-S convergiu para todas; o Stb não obteve a convergência em apenas um caso; o ILU, em dois casos e o DPA-G não convergiu para cinco matrizes. Desta forma, para esse conjunto de matrizes, os precondicionadores avaliados podem ser classificados, do mais para o menos robusto em termos de convergência, na seguinte ordem: DPA-S > Stb > ILU > DPA-G.

O GMRES com o precondicionador DPA-G convergiu para 12 das 17 matrizes apenas com 1 iteração. Acreditamos que esse resultado se deve ao esquema de engrossamento adotado, já que neste caso, no nível mais grosseiro, o GMRES como relaxador calcula o erro até que a tolerância de $10^{-8}$ seja atingida. Na maioria dos casos onde o DPA-G não convergiu, o algoritmo de matching não obteve boa redução na ordem do problema após um determinado nível. Como consequência foi gerado um alto índice de variáveis não pareadas pelo matching, não reduzindo satisfatoriamente a ordem da matriz original nos níveis mais grosseiros e prejudicando a convergência do relaxador GMRES.

A expressiva redução em número de iterações do método de solução com o uso de precondicionadores AMG não está diretamente relacionada com a melhor eficiência quando comparado ao uso do precondicionador ILU, uma vez que a iteração do GMRES com o precondicionador ILU é, em geral, muito mais rápida (em tempo computacional) que a iteração com os precondicionadores AMG.

Os melhores tempos computacionais foram alcançados pelo precondicionador DPA-G, seguido pelo ILU, Stb e, por último, o DPA-S. Em média, o DPA-G reduziu o tempo de execução em $46.19 \%$ em relação ao ILU, $46.62 \%$ em relação ao Stb e $49.92 \%$ em relação ao DPA-S.

\section{Conclusões}

Neste trabalho introduzimos o uso do método Multigrid Algébrico como precondicionador do método GMRES. Especificamente, enfatizamos o uso da estratégia de engrossamento por agregação dupla de pares (DPA) [11], comparando-a às estratégias clássicas propostas em [13] e à fatoração LU incompleta [14]. Os resultados computacionais obtidos indicam a superioridade dos métodos Multigrid avaliados em relação ao ILU. Em termos de tempo computacional, o uso desses métodos melhorou os tempos obtidos pelo ILU em 13 das 17 matrizes. E em 12 dessas 13 matrizes, a melhora foi obtida pelo uso do Multigrid com o DPA. Em termos de número de iterações, o DPA$\mathrm{G}$ convergiu com apenas 1 iteração para 12 matrizes, enquanto que, em sua maioria, os outros precondicionadores necessitaram de um número muito maior de iterações.

\section{Agradecimentos}

O presente trabalho foi realizado com apoio da Coordenação de Aperfeiçoamento de Pessoal de Nível Superior - Brasil (CAPES) - Código de Financiamento 001. Além disso, agradecemos ao Núcleo Avançado de Computação de Alto Desempenho (NACAD) da COPPE/UFRJ pela disponibilização do supercomputador Lobo Carneiro (LoboC).

\section{Referências}

[1] A. Brandt, S. McCormick e J. Ruge, Algebraic multigrid (AMG) for automatic multigrid solutions with application to geodetic computations, Report, Institute for Computational Studies, CO, 1982. 
[2] A. Brandt, General highly accurate algebraic coarsening schemes, em Proceedings of the Ninth Copper Mountain Conference on Multigrid Methods, Copper Mountain, Citeseer, 1999.

[3] W. L. Briggs, V. E. Henson e S. F. McCormick, A multigrid tutorial. Siam, 2000, vol. 72.

[4] T. A. Davis e Y. HU. The University of Florida sparse matrix collection. ACM Transactions on Mathematical Software (TOMS), 38:1-25, 2011.

[5] H. G. de Jesus, Precondicionador multigrid algébrico para métodos iterativos não estacionários na solução de sistemas lineares de grande porte, Mestrado em Informática, UFES, Vitória, 2021.

[6] R. Diestel, Graph Theory. Springer Berlin Heidelberg, 2017, vol. 173.

[7] P. W. Hemker, A note on defect correction processes with an approximate inverse of deficient rank, Journal of Computational and Applied Mathematics, 8:137-139, 1982.

[8] A. McAdams, E. Sifakis e J. Teran, A parallel multigrid Poisson solver for fluids simulation on large grids, em Proceedings of the 2010 ACM SIGGRAPH/Eurographics Symposium on Computer Animation, Eurographics Association, 2010, pages 65-74.

[9] S. F. McCormick, Multigrid methods. SIAM, 1987.

[10] SGI ICE-X. NACAD, Rio de Janeiro, 2021. Disponível em: http://www.nacad.ufrj.br/pt/recursos/sgiicex. Acesso em: 14 de junho de 2021.

[11] Y. Notay, Aggregation-based algebraic multilevel preconditioning, SIAM journal on matrix analysis and applications, 27:998-1018, 2006.

[12] J. Park, M. Smelyanskiy, U. M. Yang, D. Mudigere e P. Dubey, High-performance algebraic multigrid solver optimized for multi-core based distributed parallel systems, em Proceedings of the International Conference for High Performance Computing, Networking, Storage and Analysis, ACM, 2015.

[13] J. W. Ruge e K. Stüben, Algebraic multigrid, em Multigrid methods, SIAM, 1987, pages $73-130$.

[14] Y. Saad, Iterative Methods for Sparse Linear Systems. Minneapolis: Society for Industrial e Applied Mathematics, 2003.

[15] H. De Sterck, U. M. Yang e J. J. Heys, Reducing complexity in parallel algebraic multigrid preconditioners, SIAM Journal on Matrix Analysis and Applications, 27:1019- 1039, 2006.

[16] K. Stuben, A review of Algebraic Multigrid, CMD Report, 1999.

[17] Algebraic multigrid (AMG): an introduction with applications, Multigrid, 2000.

[18] K. Stüben, A review of algebraic multigrid, em Numerical Analysis: Historical Developments in the 20th Century, Elsevier, 2001, pages 331-359.

[19] U. Trottenberg, C. W. Oosterlee e A. Schuller, Multigrid. Elsevier, 2000.

[20] P. Vaněk, J. Mandel e M. Brezina, Algebraic multigrid by smoothed aggregation for second and fourth order elliptic problems, Computing, 56:179-196, 1996.

[21] U. M. Yang, Parallel algebraic multigrid methods - high performance preconditioners, em Numerical solution of partial differential equations on parallel computers, Springer, 2006, pages 209-236. 